\title{
1996-2016: Two decades of econophysics: Between methodological diversification and conceptual coherence
}

\author{
C. Schinckus ${ }^{\mathrm{a}}$ \\ School of Business, University of Leicester, University Road, Leicester, UK
}

Received 6 April 2016 / Received in final form 15 September 2016

Published online 22 December 2016

\begin{abstract}
This article aimed at presenting the scattered econophysics literature as a unified and coherent field through a specific lens imported from philosophy science. More precisely, I used the methodology developed by Imre Lakatos to cover the methodological evolution of econophysics over these last two decades. In this perspective, three co-existing approaches have been identified: statistical econophysics, bottom-up agent based econophysics and top-down agent based econophysics. Although the last is presented here as the last step of the methodological evolution of econophysics, it is worth mentioning that this tradition is still very new. A quick look on the econophysics literature shows that the vast majority of works in this field deal with a strictly statistical approach or a classical bottom-up agent-based modelling. In this context of diversification, the objective (and contribution) of this article is to emphasize the conceptual coherence of econophysics as a unique field of research. With this purpose, I used a theoretical framework coming from philosophy of science to characterize how econophysics evolved by combining a methodological enrichment with the preservation of its core conceptual statements.
\end{abstract}

\section{Introduction}

Econophysics is nowadays well-known as an area of knowledge dealing with the application of physics to economic/financial issues. As the name suggests, econophysics is a hybrid discipline that can roughly be defined as "a quantitative approach using ideas, models, conceptual and computational methods of statistical physics" applied to economic and financial phenomena [1]. The term "econophysics" was first coined 20 years ago by physicists by Stanley et al. [1] and since then, it has generated a lot of researches and an increasingly diversified literature [2-4]. This article aims at reconciling this methodological diversity with the idea that econophysics can be perceived as a conceptual coherent field. So doing, I will not write here a review of literature ${ }^{1}$ but I will rather propose a conceptual framework inspired

\footnotetext{
a e-mail: cs354@le.ac.uk

1 See Chakraborti [2,3] Schinckus [4] or Jovanovic and Schinckus [5] for such as review.
} 
from philosophy of science to show how econophysics literature dealing with several approaches such as agent-based econophysics (bottom-up agent-based econophysics and top-down agent-based econophysics) and statistical econophysics ${ }^{2}$ can be presented as an unique field. Specifically, this article will discuss the methodological foundations of each methodological perspective and how these approaches contributed to a progressive and coherent crystallization of econophysics.

\section{The original econophysics}

Econophysics did not pop up from nowhere: physicists who decided to deal with financial markets perpetuated a methodological trend initiated by the Santa Fa Institute in the 1990s. Indeed, as Waldrop (1992) explained it, this institution largely contributed in the study of dynamic complexity (i.e. dynamics of non-simple systems composed by a high number of interacting elements): while studies exists on the historical influence of SFI on the development of agent-based modelling (see Arthur [6] for a good overview), links with econophysics are, at best, assumed but rarely analyzed. Although that SFI is not often mentioned in the literature dedicated to econophysics, it played a key role in the genesis and the development of that field [7]. Developed and directed by well-known physicists, the Santa Fe Institute quickly became a famous research center in physics whose reputation was (is still) founded on the interdisciplinary perspective it promotes. In the 1980s, the SFI was the first place where physicists did research associated with economic systems [8]. The rapid expansion of computers contributed to the development of complexity studies because their increasing storage capacity eased the historical data analysis which favoured the identification of macro-patterns [7]. This progressive computerization of society and science contributed to the development of the self-criticality theory $[9,10]$, a field that requires a high number of observations to characterize the macro-regularities in complex systems. By promoting the application of this theory to other area of knowledge, the SFI played a key role in the genesis of econophysics. Moreover, the SFI provided an institutional place where physicists can discuss the application of that framework to economic/financial systems. This link implies that, from the statistical physicists' viewpoint, power laws are synonym to complex system, which makes their study particularly stimulating for these scientists as the leading econophysicist Bouchaud [11, p. 105] explained it,

"Physicists are often fascinated by power laws. The reason for this is that complex, collective phenomena give rise to power laws which are universal, that is, to a large degree independent of the microscopic details of the phenomenon. These power laws emerge from collective action and transcend individual specificities. As such, they are unforgeable signatures of a collective mechanism".

As the next section will detail it, this point is important because, by dealing with power laws, physicists did not deal with classical economic theoretical constraints in terms of methodological individualism, agents' perfect rationality, the Gaussian framework, arbitrage, etc. This particular methodological orientation gave econophysicists the opportunity to develop a specific knowledge outside of classical economics and, at the same time, to adopt a boundary macro-perspective on financial data. These dissimilarities with classical economics have furthermore been enhanced by the nomination of an economist (Brian Arthur) as the director of the economic program of the SFI. Indeed, this situation directly contributed to the development of

\footnotetext{
${ }^{2}$ See Schinckus [4] for a classification and a detailed review of the main works published in these sub-categories of econophysics.
} 
a specific research agenda more focused on agent-based models whose increasing popularity coincided with a gradual disinterest of works devoted to statistical patterns in economics $[12$, p. 9$]$. This historical reason partly explains why statistical patterns became an independent field of research often associated to what we call "stylized facts" in the economics. These stylized facts refer to "empirical facts that arose in statistical studies of financial (or economic) time series and that seem to be persistent across various time periods, places, markets, assets, etc." [2, p. 994] I evoke hereafter a non-exhaustive list of works dealing with two specific stylized facts (fat-tails and auto-correlations) as a telling example of this literature.

The fat-tailed dimension of financial distribution is probably the oldest ${ }^{3}$ stylized fact in finance and it is related the origin of econophysics. Indeed, since the beginning of the 1990s, this specific issue generated a great number of econophysical studies developed specific statistical frameworks such as gradually truncation [16], exponentially truncation [17], and damped truncation [18] in order to capture the leptokurticity observed in the empirical distributions. Correlations and autocorrelations between economic/financial variables (i.e. stock prices, returns, order signs, transaction volumes, etc.) can also be looked on as another stylized fact studied by the econophysicists. The Gaussian framework used in the financial mainstream implies an absence of autocorrelation for independent and identically distributed financial returns meaning that their statistical features do not depend on the time scale. However, empirical data show that statistical properties of distributions change with time. The most well-known changing characteristic is the clustering volatility which refers to the fact that "large changes tend to be followed by large changes of either sign and small changes tend to be followed by small changes" [19, p. 994]. Among the numerous works dealing with stylized facts, one can mention $[20,21]$ who tried to describe this clustering phenomenon by using specific power laws and stable Lévy processes. Because the complex phenomena require an analysis of all statistical links between elements, the econophysics literature has contributed to this specific issue related to the estimation of the correlations and covariance matrix. Stanley et al. [22] emphasized the statistical similarities (in terms of correlated randomness) between physics and economics whereas Preis et al. [23] deepened these similarities by showing that the average correlation among the daily closing prices of the 30 stocks forming the Dow Jones Industrial Average varies in time. Laloux et al. [24] found that only a few eigenvalues survive above the noise bands while several methods have been proposed for identification of the non-random elements of the correlation matrix $[25,26]$. Of course, results coming from the original correlation matrix differ strongly from those generated from the usual correlation matrix used in finance developed by Markowitz ${ }^{4}$ in 1952 [27]. As Lux [28, p. 23] explained it, "standard covariance matrix estimates might vastly overstate the chance of diversification so that better performance could be expected from using cleaned up matrices".

\footnotetext{
${ }^{3}$ Mitchell [13] for example, recognized the presence of kurtosis in financial return in 1915. However, this issue has not been studied, in finance, until the 1960s when Mandelbrot tried to describe the evolution of financial prices with a Paretian law (Pareto who pioneered the use of power law distributions even prior their use in physics). However, Jovanovic and Schinckus [14] explained why the use of a Paretian framework was not possible in finance at that time. For further detail about the use of power law in economics and social sciences, see also Gabaix [15].

4 The portfolio theory is the foundational theory of financial economics explaining how investors try to maximize portfolio expected return (i.e mean) for a given amount of portfolio risk (i.e variance), or equivalently, how investors try to minimize risk (variance) for a given level of expected return (mean), by choosing specific proportions of various assets.
} 
The econophysics literature dealing with this stylized facts is huge and its presentation goes far beyond the scope ${ }^{5}$ of this article whose purpose is to propose a conceptual framework in order to lay the methodological foundations of econophysics.

\section{The emergence of agent-based econophysics}

Schinckus [7] emphasized the important role played by the Santa Fe Institute in the popularization of dynamic complexity by introducing its two major computational approaches: the emergence of a spontaneous order (agent-based modelling) or the emergence of a macro-statistical regularity (statistical perspective). These two ways of modelling largely influenced the progressive diversification of econophysics literature which can be divided into two areas, one dealing with macro-statistical regularities and another devoted to agent-based econophysics. Around the 2000s, one can observe an increasing demand for a microscopic approach in econophysics whose original methodology was considered by some physicists (Farmer [29], Sornette [30]) as too phenomenology oriented. In this challenging context, some key econophysicists ([29-31]) promoted the creation of a methodological bridge between agent-based modelling and statistical perspective originally used in econophysics ${ }^{6}$. Although this conceptual combination between a macro-based and inductive approach (observation of statistical patterns) and a micro-based and deductive method (agent-based modelling) could be seen contradictory, it directly results from the historical roots of econophysics: these two frameworks have mainly been developed by members of the Santa Fe Institute who promoted the idea of a big science combining micro and macro studies of complex systems [33].

Agent-based econophysics is founded on a micro-approach since it uses agent-based modelling. The literature about the agent-based models is huge and published in several disciplines ${ }^{7}$. This modelling can be looked on as an interdisciplinary approach [40] referring to so many fields that it is not possible to number them in this paper. The literature dealing with agent-based models coming from physics but applied in economics can be decomposed into two categories: on one hand, we have research characterizing the emergence of specific macro-properties without using a pre-defined macro-pattern, and on the other hand, one can find works whose objective is to reproduce existing macro-statistical patterns that are taken as granted from empirical observations. It is worth mentioning that the first approach is quite similar to the one used by economists i.e. a modelling to characterize the emergence of a specific macro-result (without a priori information about the emerging macro-output) in which all micro-interactions are defined through plausible assumptions. Regarding this bottom-up agent-based modelling, one can mention the existing of several methodologies based on different analogies. For instance, the analogy "agent = particle" is usually used to describe the non-trivial behaviour of economic actors. In this perspective, herding behaviour generates several studies [41-43] associating the information dissemination process with a percolation model among traders whose interactions randomly connected their demand through clusters. Another telling

\footnotetext{
${ }^{5}$ For further details on this category of works, see Chakraborti [2] or Schinckus [3].

6 See, for example, the recent publication of a book entitled "Agent-based econophysics" at Springer Press - Abergel et al. [32].

7 Agent-based approach appeared in the 1990s as a new tool for empirical research in a lot of fields such as economics [34], voting behaviors [35], military tactics [36], organizational behaviors [37], epidemics [38], traffic congestion patterns [39], etc. See Waldrop [33] for a good "pre-history" agent-based approach in physical sciences (i.e. researches which contributed to the application of agent-based modeling in physics).
} 
examples can be mentioned with Pickhardt and Seibold [44], for example, explained that income tax evasion dynamics can be modelled through an "agent-based econophysics model" based on the Ising model of ferromagnetism while Donangelo and Sneppen [45], as well as Shinohara and Gunji [46], approached the emergence of money through studying the dynamics of exchange in a system composed of many interacting and learning agents. Another set of works implement a different analogy in their agent-based methodology since they associate physical particles with orders on the financial markets. Bak et al. [50] used a reaction diffusion model in order to describe the orders dynamics. In this model, orders were particles moving along a price line, and whose random collisions were seen as transactions (see also Farmer et al. [47] or Hommes [48], for the same kind of model). Maslov [49] tried to make the model developed by Bak et al. [50].

The second approach using agent-based technique in econophysics try to reproduce existing data implying that all micro-interactions between agents are initially calibrated in order to generate a pre-existed (given) macro-pattern. This approach is usually called "top-down agent based modelling" econophysics. In contrast with the works evoked above that do not expect any kind of macro-patterns, this topdown methodology aims at reproducing (through calibration of micro-interaction) a specific given macro-pattern. Among studies dealing with this approach, one can mention Jonhson et al. [50] and Zhou and Sornette [52] used agent-based modelling in order to reproduce the statistical stylized fact observed in the financial markets or Podobnik et al. [53] who combined stochastic and agent-based approaches to develop a model for trading time, trading volume and prices changes. Gubiec and Kutner [54] also showed that an objective mechanism can be deducted in the frame of the offer stochastic process which is independent of agents' intelligence (suggesting that statistical models can also deal with not only zero-intelligent agents).

To sum up this section, there are roughly speaking, three ways of conceptualizing the micro-macro interaction: 1) statistical econophysics (or the original econophysics); 2) bottom-up agent-based econophysics; and 3) top-down agent-based econophysics. In the next section, I will define a specific conceptual framework inspired by philosophy of science (Imre Lakatos) to highlight the methodological coherence of econophysics although one can consider its literature as scattered.

\section{The elaboration of a meta-methodology}

This section presents a set of criteria through which the three methodologies (statistical econophysics, top-down agent based econophysics and bottom-up agent-based econophysics) existing in econophysics will be discussed. Specifically, I will use a Lakatosian framework (developed by Imre Lakatos [55]) to present the similarities (and differences) between these approaches and then show the reasons for why these computational approaches can reasonably be associated. In other words, this sub-section introduces the common characteristics implicitly shared by the three methodological angles.

\subsection{The concept of "hard core"}

The concept of "hard core" is meaningful in history and philosophy of science since it echoes Lakatos's theory of rationality. Imre Lakatos associated a set of homogenous theories with what he called a research programme whose hard core refers to common features of the theories shared by all scientist acting in this research programme. In other words, the Lakatosian hardcore summarizes what members of a scientific 


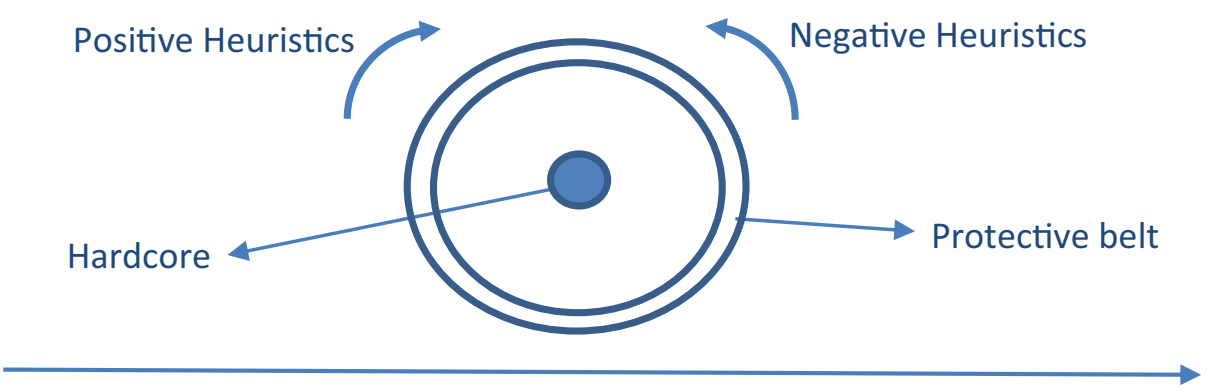

Fig. 1. Illustration of the dynamics of a Lakatosian research programme.

community take for granted in their activity. Lakatos [55] explained that these fundamental assumptions that composed the hard core are usually protected by what he called a "protective belt" (i.e. features of theories that may be altered in the research). This protective belt opens a door for the evolution of the research programme since it may evolve in line with a positive heuristic (i.e. specific plan/research agenda) exploring new issues/puzzles and their formulations required to preserve the hard core statements. This positive heuristic can be seen as a sequence of injunctions not to change the fundamental assumptions. For Lakatos, a research programme is said to be progressive when its alterations allow its members to make novel predictions which refers to the ability of the programme to predict a phenomenon that has never been observed prior the prediction. This idea of empirical progress is very important for the Hungarian philosopher because it offers a demarcation between progressive and degenerative research programmes in science. The latter characterizes a programme whose alterations are not more than ad-hoc adjustments/reformulation of the existing protective belt to preserve the hard core assumptions). Roughly speaking, the dynamics of a research programme can be illustrated as shown in Figure 1.

This schema shows that a progressive research programme is supposed to evolve in line with an increasing empirical progress (the horizontal arrow). This is the role of the positive heuristic (arrow going the same direction than the horizontal one) to ensure this specific movement. On this illustration, the arrow going in opposite direction represents the regression of the research programme in case of scholars do not follow the negative heuristic (i.e. injunctions that members must follow in order not to break the progress of the research programme). In this chapter, I will use this idea of hard core to describe the methodological fragmentation of econophysics. More precisely, I will show how the three traditions share the same hard core but that they investigate/protect it in a different way. In this context, I will suggest that the methodological diversity observed in econophysics actually result from a crystallization of the protective belt in accordance with a positive heuristic. In other terms, all econophysics will continue to share the same common feature whatever the way they will protect these fundamental assumptions. The methodological exploration made by these authors paradoxically strengthen the protective belt of econophysics as I will explain in the following section.

\subsection{The hard core of econophysics}

First of all, the three econophysics traditions deal with the extension of knowledge coming from physics to economics/finance. In this extension, the vast majority of econophysicists consider economic/financial phenomena as complex systems composed by a large number of interacting elements. This methodological point is 
important because the multiplication of components requires a specific process of generalization in order to transform the accumulation of facts/statements into a structured knowledge. While economists are well-known to found their models on the methodological individualism using the mere addition as process generalizing their knowledge 8 , econophysicists rather consider that "something happens" between the micro and the macro level of complex systems. Indeed, econophysicists conjointly consider that macro-results can be presented as an emergent properties transcending the micro-components' behaviour. However, this emergence of macro-results must be captured/explained leading each tradition to describes "what is happening" in a different way. There is an obvious link here with the philosophical concept of emergence which is usually presented as some variant of the following statement: a theory $\mathrm{T}_{1}$ reduces a theory $T_{2}$ if the laws of $T_{1}$ are derivable from those of $T_{2}$. I will show in this section that econophysics developed a different meaning for the term "reduction" since they rather associate this concept to the asymptotic convergence resulting from a large number of implementations of the reduced theory $\mathrm{T}_{2}$. This way of conceptualizing emergence can be roughly be schematized as follows:

$$
\lim _{n \rightarrow \infty} T_{2}=t_{1}
$$

In this schema, a more encompassing (macro) theory $\mathrm{T}_{1}$ reduces a specific (micro) theory $T_{2}$ if the laws of $T_{1}$ is asymptotically derived from the observations/iterations of $\mathrm{T}_{2}$. This way of characterizing the notion of emergence is inspired from Batterman [56] who promoted the development of an "asymptotic reasoning" [56, p. 3] by claiming that many of why-questions based theories are explanatorily deficient to understand how universality can arise (by universality, Batterman refers to "a feature of the world - namely that is in certain circumstances distinct types of systems exhibit similar behaviors" [56, p. 9]). When he presented his approach, the philosopher wrote that "Sometimes, science requires methods that eliminate both details and, in some sense, precision [... I I call these methods 'asymptotic methods' " [56, p. 13]. Roughly speaking the latter can be defined as methods describing limiting behaviour of a specific phenomenon. These techniques assumed the existence of a sequence of data related to a particular configuration of systems composed by noisy elements/variables. In such a context, only the asymptotic domain (behaviour at the limit-situation) is considered as a worthy information to understand the emergence of universality because it avoids details that could obscure the understanding of the phenomenon [59]. In other words, the asymptotic reasoning is appropriate to characterize a behavioural similarity observed in diverse systems (i.e. universality). It is worth mentioning that econophysicists often characterize this universality ${ }^{9}$ through the use of specific statistical processes (power laws) to describe the dynamics of complex systems. In this perspective, the universality of these processes is justified through two aspects: 1) the recurrent observation of such as regularities in different economic/financial contexts and 2) the statistical stability of the processes that guarantee a dimensionless invariant feature in the observation of complex systems. The identification of such invariance required a high number of observations/iterations whose accumulation will make emerge a recurrent macro-pattern. In other words, econophysics is based on what Batterman [56] call an asymptotic reasoning that aims

\footnotetext{
8 For more details on this issue, see Butterfield [57,58].

9 The term "universality" does not necessary have good press in philosophy of science where some authors (Berry [60, p. 185]) associate this notion with "the slightly pretentious way in which physicists denote identical behaviour in different systems". See Batterman [56] for further details about such as systems exhibiting the same macro-behaviour while we obviously know that their micro-details differ significantly.
} 
Table 1. The hardcore of econophysics.

\begin{tabular}{|l|l|}
\hline \multicolumn{2}{|c|}{ The hard core of econophysics } \\
\hline Area of knowledge & Economic/financial phenomena \\
\hline Explanandum & $\begin{array}{l}\text { Emergent properties based systems (interaction } \\
\text { between the micro and the macro level) }\end{array}$ \\
\hline Explanans & By using an asymptotic reasoning \\
\hline
\end{tabular}

at formulating equations describing universal features ( $\Delta$ for instance) of systems by assuming that the limits $\Delta_{i}(\infty)$ exist.

In line with this reasoning, econophysicists from all traditions assume that complex phenomena can be captured through the analysis of a high number of observations/iterations. The idea behind an asymptotic reasoning is to develop a method that eliminates micro-details to highlight a significant regularity echoing to an identical asymptotic behaviour in different systems/phenomena. As evoked above, this regularity often takes the statistical form of a power law presented as a "universal feature" and it is justified by the use of asymptotic statistics in order to eliminate irrelevant details about individual elements. Although the three econophysics traditions are founded on an asymptotic reasoning, it is worth mentioning that they use different techniques to characterize this asymptotic modelling. The next section will explain in more details how statistical econophysics, bottom-up agent-based econophysics and top-down agent-based econophysics implement this asymptotic reasoning. At this step of the article, the point is to understand that the use of an asymptotic reasoning is a part of the conventionally accepted statements among all econophysics that can be summarized as shown in Table 1.

These core elements define the intellectual scope of econophysics and they describe the major paths of research pursued by econophysicists. First of all, it is commonly accepted that econophysics refers to the extension of physics to finance and economics. That being said, econophysics agree on what has to be modelled (explanandum) since all of them consider emergent properties based systems as the target of their research [19]. Facts and statements about these systems can then be observed through a specific way of modelling (explanans) based on an asymptotic reasoning according to which a high number of observations/iterations can reveal a knowledgeable statement even though it is based on a fictional element referring to a passage to the limit.

\subsection{Methodological diversification and conceptual coherence}

The purpose of this article is to show that the evolution of econophysics implying a methodological diversification does not lose its original hard core. With this purpose, I will consider the development of a Lakatosian research programme as a multi-layered process that can take several forms [61]. According to Lakatos, this development suggests an empirical progress characterized by the observation of novel predictions. Because these novel predictions allows scholars to outline unknown phenomena, such as view of scientific progress refers to an increasing of advancement of scientific knowledge (cognitive progress). However, this way of describing the enrichment of knowledge mainly focuses on the goal of a research programme but it underestimates other aspects of scientific progress ${ }^{10}$ that can also be expressed in a more technological (increased effectiveness of techniques), social increasing quality of life and justice in society), professional (rising status of the scientific institutions) or methodical (invention of new method of research) forms [61]. This paper deals

10 For more details about these debates, see Niiniluoto [61]. 


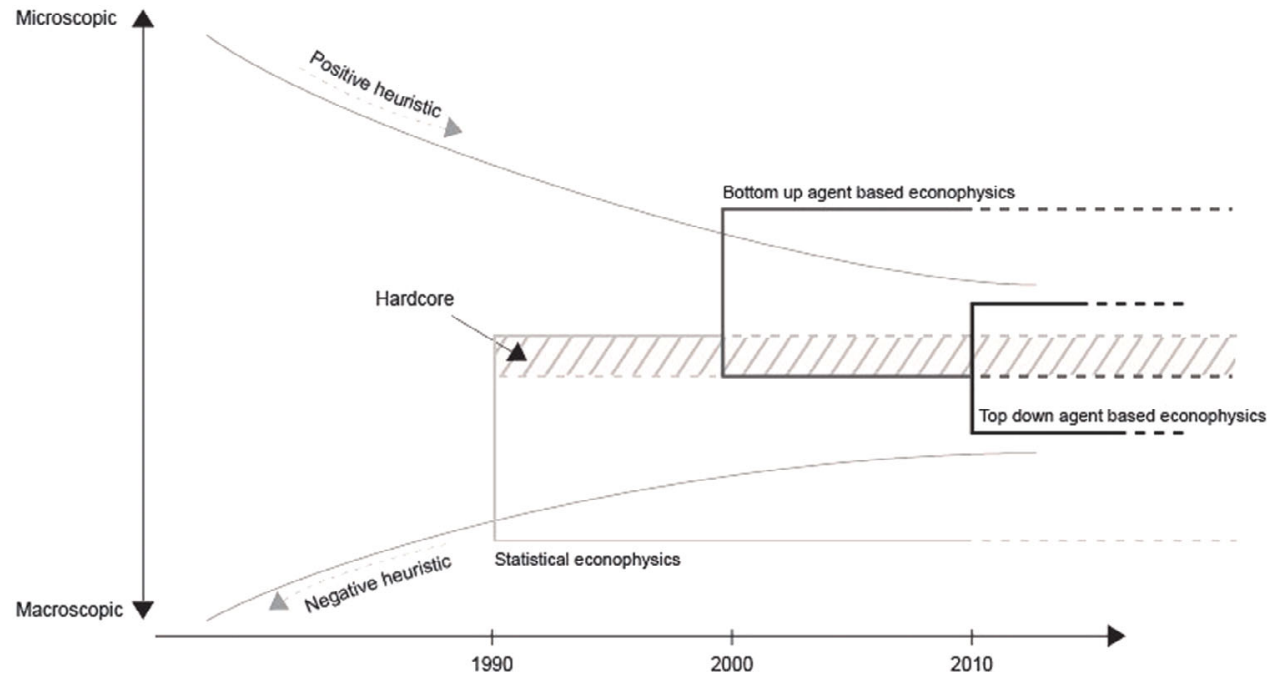

Fig. 2. Illustration of the methodological evolution of econophysics.

with the last dimension since, it will use this idea of a multi-layered development of research programme to show that the proliferation of econophysics methodologies results from a specific evolution of the protective belt (i.e. assumption protecting the hard core) induced by can the positive heuristic of the field.

Two aspects of the research programme evolution will be studied here. First of all, I acknowledge that the three econophysics traditions evoked above keep the same conceptual hard core and the same major objective which is to make predictions regarding phenomenon (emergent properties based systems) whose mechanism appears for a long time as unknown for scholars (cognitive dimension). The second aspect I would like to develop here concerns the methodical evolution of a scientific enterprise. By keeping its core statements identified in the previous section while improving the way of describing financial/economics issues, econophysics generated a diversification of scientific instruments that led to the methodological diversification. While the original econophysics focused on the statistical description of economic/financial systems without dealing with their individual components, other econophysicists explored some methodological paths based on computer simulations. So doing, they contributed to the methodical development of econophysics that can be schematized as shown in Figure 2.

This figure schematizes the evolution of econophysics through two dimensions: cognitive (horizontal axis referring to the advancement of knowledge regarding emergent phenomena) and methodical (vertical axis describing the refinement of instrument used in econophysics). While the first rectangle describes the original (statistical) econophysics that mainly uses a macroscopic based approach, the second rectangle refers to a corpus of works founded on more a microscopic perspective and, in this perspective it illustrates the bottom-up agent-based econophysics that emerged in the 2000's. Finally the last rectangle on this Figure 2 is associate with the top down agent-based econophysics. This graph shows that the proliferation of approaches results from a specific evolution of econophysics in accordance with a positive heuristic whose objective was to solidify key assumptions (i.e. protective belt) of the field by keeping its hard core unmodified. As mentioned before, the positive heuristic consists of articulated set of suggestions on how to change and solidify the protective belt as Lakatos explained it. 
"The positive heuristics sets out a programme which lists a chain of ever more complicated models simulating reality: the scientists' attention is riveted on building his models following instructions which are laid down in the positive part of his programme. He ignores the actual counterexamples" (Lakatos [55, p. 50]).

In other words, the positive heuristic gives the members of research programme the opportunity to investigate more complicated models without calling the hard core of the field into question. The use of the word "model" is interesting because it opens a door to the methodological aspect that I mentioned above. Indeed, when Lakatos refers to the concept of "model" in his definition of the positive heuristic, he means "a set of initial conditions (possibly together with the observational theories) which one know is bound to be replaced during the further development of the programme" (Lakatos [55, p. 51]).

By considering the methodological aspect (i.e. refinement of instruments), I directly illustrate this potential replacement of initial condition evoked by Lakatos. While original (statistical) econophysics used a macroscopic approach with its specific initial conditions (high number of observation), the bottom-up agent based econophysics that emerged several years later rather focuses on a microscopic perspective implying different initial conditions based on the pre-definition of the microinteraction between components. Finally the last tradition (top down agent-based econophysics) provides a modelling requiring initial conditions that combined the ones used by the two other approaches.

\section{Conclusion}

After two decades of research, econophysics is dynamic area of knowledge generating a lot of works combining different methodical approaches. In their investigation, econophysics dared to explore new methodological paths leading to a diversification of the literature. In this context of diversification, the objective (and contribution) of this article is to emphasize the conceptual coherence of econophysics as a field of research. With this purpose, I used a theoretical framework coming from philosophy of science to characterize how econophysics evolved by combining a methodological enrichment with the preservation of its core conceptual statement (i.e. statement implicitly shared by the majority of econophysicists). Although this article mainly focuses on the conceptual coherence of the field, the discussion proposed in the paper also contributes to a better understanding of dissimilarities that exist between the co-existing methodological approaches in econophysics. Roughly speaking, these differences can be summarized as shown in Table 2 .

The first line of Table 2 refers to the methodological angle chosen by the three methodological traditions to deal with complex economic/finance systems. The second one echoes to the initial conditions (i.e. starting points) these traditions required to implement their methodology. Afterwards, I evoke the form of knowledge these three approaches proposes by clarifying the outputs they offer. This table also differentiates these traditions in terms of goal and machinery (techniques) used by econophysicists. The goals to what scholars want to do by implementing their research. Their goal can be backward-looking or forward-looking since it can consider the present as the starting or the final point of the research. While statistical econophysics aims at fitting historical data for description (backward-looking, the bottom up agent based econophysics rather algorithmically reproduce data for predictive purpose (forwardlooking). Finally, the last element of my meta-methodology refers to the way of characterizing the concept of emergence in the three econophysics approaches. This article 
Table 2. The three traditions in econophysics.

\begin{tabular}{|c|c|c|c|}
\hline & $\begin{array}{l}\text { Statistical } \\
\text { Econophysics }\end{array}$ & $\begin{array}{l}\text { Bottom-up } \\
\text { agent based } \\
\text { Econophysics }\end{array}$ & $\begin{array}{l}\text { Top-down } \\
\text { agent based } \\
\text { Econophysics }\end{array}$ \\
\hline Methodology & Phenomenological & Bottom-up & Top-Down \\
\hline Initial conditions & $\begin{array}{l}\text { High number } \\
\text { of observations }\end{array}$ & $\begin{array}{l}\text { Pre-defined } \\
\text { micro-interactions }\end{array}$ & $\begin{array}{l}\text { Statistical } \\
\text { assumptions }\end{array}$ \\
\hline Outcomes & $\begin{array}{l}\text { Statistical } \\
\text { macro-patterns }\end{array}$ & $\begin{array}{l}\text { Emerging } \\
\text { macro-order }\end{array}$ & $\begin{array}{l}\text { Micro-interactions } \\
\text { compatible with } \\
\text { pre-existing } \\
\text { a macro-pattern }\end{array}$ \\
\hline Goal & $\begin{array}{l}\text { Backward-looking } \\
\text { (Fitting data for } \\
\text { descriptive } \\
\text { purpose) }\end{array}$ & $\begin{array}{l}\text { Forward-looking } \\
\text { (Reproducing data } \\
\text { for } \\
\text { predictive } \\
\text { purpose) }\end{array}$ & $\begin{array}{l}\text { Combination of } \\
\text { fitting and reproduc- } \\
\text { ing data for predictive } \\
\text { purpose }\end{array}$ \\
\hline Machinery & Statistical processes & $\begin{array}{l}\text { Algorithmic } \\
\text { processes }\end{array}$ & $\begin{array}{l}\text { Statistical/ } \\
\text { Algorithmic processes }\end{array}$ \\
\hline Emergence & $\begin{array}{l}\text { No condition } \\
\text { of derivability } \\
\text { between the } \\
\text { macro and the } \\
\text { micro level }\end{array}$ & $\begin{array}{l}\text { Definition of the } \\
\text { micro level from } \\
\text { which the macro } \\
\text { level must be } \\
\text { derived }\end{array}$ & $\begin{array}{l}\text { Definition of the } \\
\text { macro level to define } \\
\text { the micro level must } \\
\text { be derived }\end{array}$ \\
\hline
\end{tabular}

identified a conceptual hard core for these three methodologies by showing how this hard core is not altered in the methodological diversification of econophysics.

\section{References}

1. H. Stanley, V. Afanasyev, L.A.N. Aamaral, S.V. Buldyrev, A.L. Goldberger, S. Havlin, H. Leschhorn, P. Maass, R. Mantegna, C.-K. Peng, P.A. Prince, M.A. Salinger, M.H.R. Stanley, G.M. Viswanathan, Anomalous fluctuations in the dynamics of complex systems: from DNA and physiology to econophysics, Physica A 224, 302 (1996)

2. A. Chakraborti, I. Muni Toke, M. Patriarca, F. Abergel, Econophysics review: I. Empirical Facts, Quant. Finance 11, 991 (2011)

3. A. Chakraborti, I. Muni Toke, M. Patriarca, F. Abergel, Econophysics review: I. Agentbased Models, Quant. Finance 11, 1013 (2011)

4. C. Schinckus, Statistical econophysics and agent-based econophysics, Quant. Finance 12, 1189 (2012)

5. F. Jovanovic, C. Schinckus, Econophysics and Financial Economics: The Emergence of a Dialogue (Oxford University Press, forthcoming 2016)

6. B. Arthur, Complexity and the Economy (Oxford University Press, New York, 2014)

7. C. Schinckus, Econophysics and Complexity Studies, Ph.D. Dissertation, University of Cambridge, 2016

8. R. Mantegna, E. Stanley, An introduction to Econophysics (Cambridge University Press, New York, 1999)

9. P. Bak, M. Paczuski, M. Shubik, Price variations in a stock market with many agents, Physica A 246, 430 (1997)

10. R. Hughes, in Models as Mediators: Perspectives on Natural and Social Science, edited by M.S. Morgan and M. Morrison (Cambridge University Press, Cambridge, 1999), p. 97

11. J.-P. Bouchaud, Power laws in economics and finance: some ideas from physics, Quant. Finance 1, 105 (2001)

12. S. Durlauf, Complexity and Empirical Economics, Econ. J. 115, F225 (2005) 
13. W. Mitchell, The Making and Using of Index Numbers, Bulletin of the United States Bureau of Labor Statistics 173, 5 (1915)

14. F. Jovanovic, C. Schinckus, Towards a transdisciplinary econophysics, J. Econ. Methodology 20, 164 (2013)

15. X. Gabaix, Power Laws in Economics and Finance, Annu. Rev. Econ. 1, 255 (2009)

16. H. Gupta, J. Campanha, The gradually truncated Lévy flight for systems with power-law distributions, Physica A 268, 231 (1999)

17. H. Gupta, J. Campanha, Tsallis statistics and gradually truncated Lévy flight - distribution of an economic index, Physica A 309, 381 (2002)

18. R. Matsushita, P. Rathie, et al., Exponentially Damped Lévy Flight, Physica A 326, $544(2003)$

19. D. Rickles, in J. Collier, C. Hooker (eds.), Handbook of the Philosophy of Science: Philosophy and Complex Systems (North Holland Elsevier Editions, New York, 2008), Vol. 10

20. P. Cizeau, Y. Liu, M. Meyer, C.K. Peng, H.E. Stanley, Volatility distribution in the S\&P500 stock index, Physica A 245, 441 (1997)

21. Y. Liu, P. Cizeau, M. Meyer, C.K. Peng, H.E. Stanley, Correlations in economic time series, Physica A 245, 437 (1997)

22. E. Stanley, S. Buldyrev, G. Franzese, S. Havlin, F. Mallamace, P. Kumar, V. Plerou, T. Preis, Correlated randomness and switching phenomena, Physica A 389, 2880 (2010)

23. T. Preis, D. Kenett, E. Stanley, D. Helbing, E. Ben-Jacob, Quantifying the Behavior of Stock Correlations under Market Stress, Nat. Sci. Rep. 2, 752 (2012)

24. L. Laloux, P. Cizeau, J. Bouchaud, M. Potters, Noise dressing of financial correlation matrices, Phys. Rev. Lett. 83, 1467 (1999)

25. J.D. Noh, Model for correlations in stock markets, Phys. Rev. E 61, 5981 (2000)

26. S. Galluccio, J.P. Bouchaud, M. Potters, Portfolio optimization with short-selling and spin-glass, Physica A 259, 449 (1998)

27. H.M. Markowitz, Portfolio Selection, J. Finance 7, 77 (1952)

28. T. Lux, Applications of Statistical Physics in Finance and Economics, Working paper no 1425, University of Kiel, 2008

29. D. Farmer, Scale the Ivory Towers of Finance, Working Papers 99-10-073, Santa Fe Institute, 1999

30. D. Sornette, Why Stock Markets Crash: Critical Events in Complex Financial Systems (Princeton University Press, New Princeton, 2003)

31. R. Cont, M. Potters, J.-P. Bouchaud, in Proceedings of the CNRS Workshop on Scale Invariance (CNRS: Les Houches France, 1997)

32. F. Abergel, H. Aoyama, B. Chakrabarti, A. Chakraborti, A. Ghosh, Econophysics of Agent-Based Models (Springer, 2014)

33. M. Waldrop, Complexity:The Emerging Science at the Edge of Order and Chaos (Simon and Schuster, USA, 1992)

34. R. Axtell, The Emergence of Firms in a Population of Agents: Local Increasing Returns, Unstable Nash Equilibria, and Power Law Size Distributions, Santa Fe Institute Working Paper 99-03-019, 1999

35. K. Lindgren, M.G. Nordahl, Cooperation and Community Structure in Artificial Ecosystems, Artificial Life 1, 15 (1994)

36. A. Ilachinski, Irreducible Semi-Autonomous Adaptive Combat (ISAAC): An ArtificialLife Approach to Land Warfare, Center for Naval Analyses Research Memorandum, CRM 97-61.10, 1997

37. M. Prietula, K.M. Carley, L. Gasser, editors, Simulating Organizations: Computational Models of Institutions and Groups (AAAI Press, Menlo Park, CA, 1998)

38. J.M. Epstein, R. Axtell, Growing Artificial Societies: Social Science form Bottom Up (MIT Press, Cambridge, 1996)

39. S. Rasmussen, K. Nagel, in R. Brooks (ed.), Artificial Life IV (MIT Press, Cambridge, MA, 1994) 
40. J.M. Epstein, Generative Social Science: Studies in Agent-Based Computational Modelling (Princeton University Press, Princeton, 2006)

41. V.M. Eguíluz, M.G. Zimmermann, Transmission of information and herd behavior: An application to financial markets, Phys. Rev. Lett. 85, 5659 (2000)

42. D. Stauffer, D. Sornette, Self-organized percolation model for stock market fluctuations, Physica A 271, 496 (1999)

43. J. Wang, C. Yang, P. Zhou, Y. Jin, T. Zhou, B. Wang, Evolutionary percolation model of stock market with variable agent number, Physica A 354, 505 (2005)

44. M. Pickhardt, G. Seibold, Income Tax Evasion Dynamics: Evidence from an Agent-based Econophysics Model, Working paper of the University of Cottbus, 2011

45. R. Donangelo, K. Sneppen, Self-organization of value and demand, Physica A 110, 572 (2000)

46. S. Shinohara, Y. Gunji, Emergence and collapse of money through reciprocity, Physica A 117, $131(2001)$

47. D. Farmer, P. Patelli, I. Zovko, The predictive power of zero intelligence in financial markets, Proc. Natl. Acad. Sci. USA 102, 2254 (2005)

48. C. Hommes, Financial markets as complex adaptive evolutionary systems, Quant. Finance 1, 149 (2001)

49. S. Maslov, Simple model of a limit order-driven market, Physica A 278, 571 (2000)

50. P. Bak, M. Paczuski, M. Shubik, Price variations in a stock market with many agents, Physica A 246, 430 (1997)

51. N.F. Johnson, D. Lamper, P. Jefferies, M.L. Hart, S. Howison, Application of multi-agent games to the prediction of financial time series, Physica A 299, 222 (2001)

52. W.-X. Zhou, D. Sornette, Self-organizing Ising model of financial markets, Eur. Phys. J. B 55, 175 (2008)

53. B. Podobnik, D. Wang, E. Stanley, High frequency trading model for a complex trading hierarchy, Quant. Finance 12, 559 (2012)

54. T. Gubiec, R. Kutner, Backward jump continuous-time random walk: An application to market trading, Phys. Rev. E 82, 46 (2010)

55. I. Lakatos, The Methodology of Scientific Research Programmes: Philosophical Papers (Cambridge University Press, Cambridge, 1978), Vol. 1

56. R. Batterman, The Devil in the Details: Asymptotic Reasoning in Explanation, Reduction and Emergence (Oxford University Press, New York, 2002)

57. J. Butterfield, Emergence, Reduction and Supervenience: A Varied Landscape, Found. Phys. 41, 920 (2011)

58. J. Butterfield, Less is Different: Emergence and Reduction Reconciled, Found. Phys. 41, 1065 (2011)

59. R. Batterman, Into a mist: Asymptotic theories on a caustic, Studies in the History and Philosophy of Modern Physics 28, 395 (1997)

60. M. Berry, The Bakerian Lecture: Quantum Chaology in M. Berry, I. Percival, N. Weiss (eds.), Dynamical Chaos (Princeton University Press, Princeton, 1987), Vol. 186, p. 183

61. I. Niiniluoto, Scientific Progress in Stanford Encyclopaedia of Philosophy, http://plato. stanford.edu/entries/scientific-progress/ (2015)

Open Access This is an Open Access article distributed under the terms of the Creative Commons Attribution License (http://creativecommons.org/licenses/by/4.0), which permits unrestricted use, distribution, and reproduction in any medium, provided the original work is properly cited. 